\title{
EFECTOS DE UN PROGRAMA DE ASERTIVIDAD EN \\ ADOLESCENTES DE UNA INSTITUCIÓN EDUCATIVA PRIVADA DE VENTANILLA - CALLAO
}

\author{
Effects of an Assertiveness Program in adolescents of a Private Educational \\ Institution of Ventanilla - Callao
}

\author{
Lizley Janne Tantaleán Terrones*
}

\begin{abstract}
Resumen
La presente investigación refiere los efectos de un programa de asertividad en 40 adolescentes, entre 11 a 17 años de edad, del $1^{\circ}$ al $5^{\circ}$ año de secundaria de una Institución Educativa Privada de Ventanilla - Callao. El estudio fue exploratorio, cuasi- experimental, se utilizó el Inventario de Autoinforme de la Conducta Asertiva ADCA-1. Los resultados del test - retest evidenciaron cambios en la asertividad del total de participantes con $X 2 r$ de Pearson $=4,568$ y significación de $p<, 033$, al igual que en alumnos de $1^{\circ}$ año con $Z$ Wilcoxon $=$ $-2,278$ y $p<, 023$, así como en los de 13 años con $Z=-2,023$ y $p<, 043$; (siendo poco significativo en el $2^{\circ}$, $3^{\circ}$, $4^{\circ}$ y $5^{\circ}$ año, al igual que en los de 12, 14 y 15 años de edad). No existen cambios significativos post test, según sexo y tipo de familia analizados con la prueba U de Mann-Whitney. Por tanto se concluye que el programa ha sido efectivo en el desarrollo de asertividad del grupo analizado, con implicancias distintas según las características del mismo.
\end{abstract}

Palabras clave: Asertividad, entrenamiento asertivo, adolescentes.

\begin{abstract}
This research concerns the effects of an assertiveness program in 40 adolescents, between 11 to 17 years old, from 1st to 5th year of high school of a Private Educational Institution of Ventanilla - Callao. The study was exploratory, quasi-experimental; the Self-report Inventory of Assertive Behavior ADCA-1 was used. The results of the test - retest showed changes in the assertiveness of all participants with Pearson X2r $=4.568$ and significance of $p<, 033$, as well as in 1st-year students with Wilcoxon $Z=-2.278$ and $p<, 023$, and in 13-year-old adolescents with $Z=-2.023 p<, 043$; (being insignificant in the $2 \mathrm{nd}$, 3rd, 4th and 5th year, like in 12, 14 and 15 year-old adolescents). There are no significant posttest changes, according to sex and family type, analyzed using Mann-Whitney $U$ test. Therefore, it is concluded that the program has been effective in developing assertiveness of the analyzed group, with different implications according to its characteristics.
\end{abstract}

Keywords: Assertiveness, assertiveness training, adolescents.

\footnotetext{
* Licenciada en Psicología por la Universidad Nacional Federico Villarreal, Magíster en Psicología con mención en Prevención e Intervención en Niños y Adolescentes por la Universidad Femenina Del Sagrado Corazón, Diplomada en Gestión del Talento Humano por la Universidad ESAN. Coordinadora del Equipo Técnico del Centro de Atención Residencial San Martin de Porres - INABIF (Programa Nacional para el Bienestar Familiar) - Ministerio de la Mujer y Poblaciones Vulnerables. lizleytantalean@hotmail.com).
} 


\section{INTRODUCCIÓN}

Este trabajo de investigación se basa en el desarrollo de la asertividad, variable que comprende la capacidad de autoafirmar los propios derechos. Wolpe (1958, citado en Jara, 1997) la define como la expresión de todo sentimiento desprovisto de ansiedad (observando que la ansiedad puede actuar como inhibidora en circunstancias, en las que se necesita reconocer y saber expresar adecuadamente los estados afectivos). Por su parte Fábregas y García (1988) consideran que la conducta asertiva consiste en que la persona conozca sus derechos e intereses personales y los defienda, utilizando una serie de habilidades conductuales que le permitirán ser objetivo y respetuoso con las demás personas, y Navas (1995, citado en Jara, 1997) la asume como un estilo de comunicación e interacción que permite ejercer con firmeza y honestidad nuestros derechos de manera que no tengamos la necesidad que humillar o dominar a otras personas.

Se puede concluir entonces que la asertividad constituye un estilo de comunicación y comportamiento que permite la adaptación del individuo al ambiente y obtener recompensas a corto, mediano y largo plazo, por que asume la solución de problemas inmediatos, disminuyendo la aparición de problemas futuros en la interacción social, siendo eficaz no solo para manifestar de forma adecuada sentimientos negativos y evitar conflictos; sino también para la expresión de sentimientos de amor, agrado y afecto.

Se entrena en asertividad con la finalidad de descondicionar hábitos inadaptables de las respuestas ansiosas, ayudando a la expresión apropiada de emociones auténticas. En este caso el modelo que mejor se ajusta es el cognitivo conductual, teniendo como base las fases para el desarrollo de habilidades sociales (en una versión más evolucionada, que considera implícito el desarrollo del modelado, la retroalimentación, el reforzamiento y mantenimiento), implementando además tres formas específicas en el caso de la asertividad, entre lo que se considera el uso de técnicas de reestructuración cognitiva (dándole importancia a las creencias y los sentimientos que se asocian; así como la necesidad de reorganizar el esquema con ideas y actitudes alternativas, que generen mayor bienestar a la persona), técnicas para enfrentar discusiones y técnicas para reducir la ansiedad. Sobre las técnicas para enfrentar discusiones Castanyer (2007) propone algunas de gran utilidad, como el disco rayado, que consiste en repetir en forma calmada varias veces el mensaje que se desea emitir (utilizándola para expresar peticiones y/o rechazos), evitando así ser objeto de la manipulación de los demás; en cuanto a la técnica para reducir la ansiedad se toma en cuenta ejercicios de relajación y de respiración.

Este estudio buscó analizar los efectos de un programa para el desarrollo de la asertividad en adolescentes peruanos, por lo que es importante tomar algunos datos, sobre el contexto de estos, que representan más del $20 \%$ de la población nacional (Instituto Nacional de Estadística e Informática [INEI], 2011). A su vez la Encuesta Demográfica y de Salud Familiar publicada por el INEI (2000), indica que más del $50 \%$ de adolescentes se encuentran en condiciones de pobreza, conviviendo con otras problemáticas de índole psicosocial, como el índice de embarazo y/o maternidad que incluye al $13 \%$ de las adolescentes y un elevado porcentaje de mortandad en quienes optan por un aborto asistido. Calle (2005) afirma que estos/as adolescentes se encuentran involucrados/as en un sin número de situaciones de alto riesgo, tales como, la probabilidad del contagio VIH, debido a una conducta sexual irresponsable; el consumo de drogas lícitas e ilícitas (que ha denotado un mayor porcentaje en el consumo de alcohol seguido por el de tabaco, etc.); el ser víctimas potenciales de agresión física, psicológica, violación, acoso sexual y/o moral; desarrollar inseguridad e inestabilidad emocional, caer en depresión o intentar suicidarse, entre otros problemas vinculados a su situación educativa, socio cultural y socioeconómica, tomando en cuenta que en el Perú aproximadamente el $18.3 \%$ de los adolescentes de 15 a 19 años se dedica solo a trabajar, el $47 \%$ solo estudia, el $21 \%$ estudia y trabaja y un preocupante $13 \%$ no estudian ni trabaja

La problemática del adolescente posee diversas aristas, por lo que requiere de un análisis que identifique factores protectores precisos y adecuados que los coadyuven a superar las dificultades del día a día, disminuyendo la influencia de los factores de riesgo asociados a esa etapa de vida.

Este contexto nos expresa la gran necesidad que tienen los adolescentes de desarrollar conductas 
alternativas, para lo cual se propone un programa que busca elevar el nivel de asertividad en los adolescentes, mediante la utilización de una dinámica que los aliente a asumir una serie de cambios personales, que los favorezcan individualmente e influyan considerablemente en su entorno (Montgomery, 1997). Trabajar en esta área es provechoso para originar un cambio social, por lo que la presente investigación trata de dar sustento a un programa de asertividad aplicado a 40 adolescentes de una institución educativa privada, del distrito de Ventanilla en la provincia constitucional del Callao.

El programa se denomina "Hazte cargo de tu vida" (HCTV), y se verifica su efecto midiendo la asertividad obtenida por cada adolescente antes y después de su participación en las 8 sesiones que lo componen, sesiones que se encuentran dentro del marco del entrenamiento asertivo haciendo uso de las técnicas: de restructuración cognitiva, de afrontamiento de discusiones y para la reducción de la ansiedad (Lange, 1981; Castanyer, 2007).

\section{MÉTODO}

Es un estudio exploratorio (dada la novedad del programa aplicado) y cuasi experimental (pues se manipula la variable asertividad, sin mantener estricto control sobre la misma), en una muestra compuesta por 40 adolescentes entre 11 a 17 años de edad, estudiantes del nivel secundario de una institución educativa privada del distrito de Ventanilla - Callao, quienes asistieron al 100\% de las sesiones del programa.

El diseño del muestreo es no probabilístico - intencional, no utilizándose para ello ninguna técnica de igualación de grupos o asignación al azar (Hernández, Fernández y Baptista, 2006).

Se aplicó el programa "HCTV", que publicado en Irlanda por Quinn M. y Quinn T. en 1990, traducido al español en el Perú por el Sacerdote de la Congregación Salesiana P. Ennio Leonardi en 1994 y editada el año 2002 (versión utilizada). Consta de 8 sesiones dedicadas ampliamente a desarrollar la asertividad mediante un enfoque cognitivo conductual. Cabe indicar que fue diseñado para personas de 18 a 25 años, pero para los fines de este estudio se adaptó su contenido a una población adolescente entre 12 a 17 años de edad; para luego validarlo mediante el criterio de 6 jueces especialistas en metodología de la investigación, elaboración de programas preventivos y/o proyectos sociales de la Universidad Femenina del Sagrado Corazón (UNIFÉ), ello con el fin de valorar la claridad, así como la bondad de cada sesión, proporcionándole un lenguaje y presentación apropiadas (observar que aunque el programa inicialmente se propuso para adolescentes entre 12 a 17 años, asistieron algunos de 11 años, a todas las sesiones motivo por el cual se consideraron sus datos, para el análisis general).

Para medir los efectos del programa "HCTV", se utilizó el Inventario de Auto-informe de la Conducta Asertiva (ADCA -1), en su versión original (García y Magaz, 2005, citados en Guerrero y Lizano, 1998), mediante el método test re-test. En cuanto a la validez de la prueba los autores, consideraron el criterio de validez concurrente, correlacionando los resultados del Inventario de Auto-informe de la Asertividad ADCA-1, con los obtenidos en una escala similar: la Escala de Asertividad de Rathus. En una muestra de 264 estudiantes de 16 a 21 años, encontrando una correlación con Rathus, de -0.50, para auto-asertividad y -0.27 hetero-asertividad, Olano y Risco (2005) analizaron su confiabilidad en 201 personas entre adolescentes y adultos, estudiantes de la Universidad César Vallejo (Trujillo - Perú), obteniendo un Alfa de Cronbach, de 0.8541 para auto-asertividad y 0.8690 para hetero-asertividad, lo que indica su alta confiablilidad.

\section{RESULTADOS}

Luego de haber terminado el estudio, se muestran los resultados, los cuales se presentaron inicialmente en frecuencias y porcentajes. Ver tablas 1 y 2 , así como las figuras 1 y 2 :

Tabla 1

Niveles de auto-asertividad alcanzados antes y después, del programa asertividad en adolescentes $(\mathrm{N}=40)$

\begin{tabular}{llllc}
\hline & \multicolumn{2}{c}{ Pre test } & \multicolumn{2}{c}{ Post test } \\
$\begin{array}{l}\text { Niveles de Auto- } \\
\text { asertividad }\end{array}$ & $f$ & $\%$ & $f$ & $\%$ \\
\hline Superior & 13 & 32.5 & 22 & 55 \\
Promedio & 16 & 40 & 13 & 32.5 \\
Inferior & 11 & 27.5 & 5 & 12.5 \\
\hline
\end{tabular}


En la tabla 1 se observan las frecuencias y porcentajes de los niveles de auto-asertividad alcanzados antes y después, de la aplicación del programa, apreciándose un incremento del $69.2 \%$ en la cantidad de participantes que alcanzan el nivel superior en el pre test con respecto al post test. Concluyendo que los niveles de auto- asertividad se han elevado, como se representa en la figura 1.

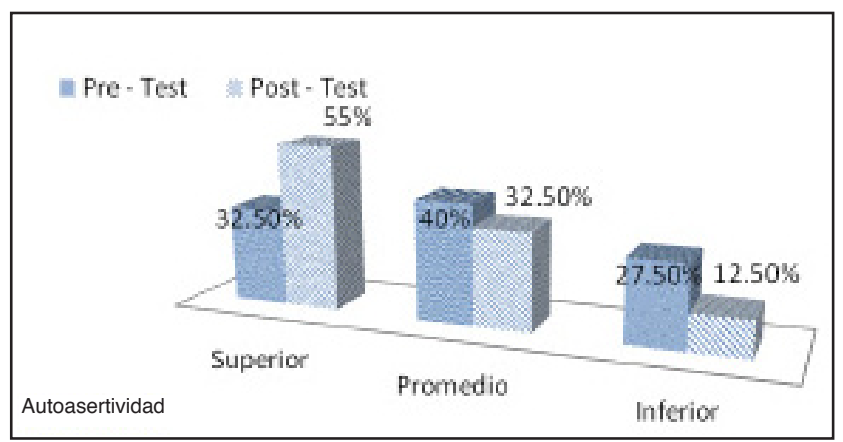

Figura 1: Diagrama de barras de los niveles de auto-asertividad pre y post test

A su vez, los niveles de hetero-asertividad alcanzados por los participantes, se indican en la tabla 2:

Tabla 2

Niveles de hetero-asertividad alcanzados antes y después, del programa asertividad en adolescentes $(\mathrm{N}=40)$

\begin{tabular}{lllll}
\hline & \multicolumn{2}{l}{ Pre test } & \multicolumn{2}{c}{ Post test } \\
$\begin{array}{l}\text { Niveles de Hetero- } \\
\text { asertividad }\end{array}$ & $\mathrm{f}$ & $\%$ & $\mathrm{f}$ & $\%$ \\
\hline Superior & 11 & 27.5 & 18 & 45 \\
Promedio & 17 & 42.5 & 15 & 37.5 \\
Inferior & 12 & 30 & 7 & 17.5 \\
\hline
\end{tabular}

En la tabla 2 se observan las frecuencias y porcentajes de los niveles de Hetero-asertividad antes y después, de la aplicación del programa, apreciándose un incremento del $63.6 \%$ en la cantidad de participantes que obtienen el nivel superior en el pre test con respecto al post test. Por tanto los niveles de
Hetero- asertividad se han elevado, como se representa en la figura 2.

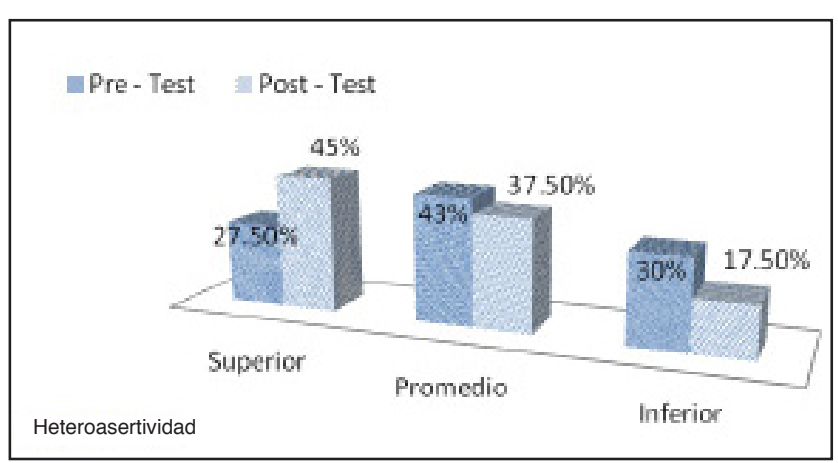

Figura 2: Diagrama de barras de los niveles de hetero-asertividad pre y post test

El análisis estadístico, se realizó con las pruebas X2rde Friedman, Z de Wilcoxon y U de Mann-Whitney, de forma independiente, según las necesidades de la investigación.

Los siguientes resultados simbolizan las diferencias antes y después de haber aplicado un programa de asertividad.

Tabla 3

Comparación pre y post test con la prueba $\mathrm{X}^{2}$ de Friedman para el ADCA - I, en adolescentes $(\mathrm{N}=40)$

\begin{tabular}{lcccc} 
& $\mathrm{N}$ & Rango promedio & $\mathrm{X} 2 \mathrm{r}$ & Nivel de significación \\
\hline Antes & 40 & 1,34 & & \\
Después & 40 & 1,66 & 4,568 &, $033^{*}$ \\
\hline
\end{tabular}

$* \mathrm{P}<.05$

En la tabla 3 se observa la comparación pre y post test de los adolescentes participantes de la investigación, con la prueba X2r de Friedman; en la cual se muestra un rango promedio de 1.34 para la medida pre test y de 1,66 para la medida post test y un puntaje X2r encontrado de 4,568 con un nivel de significancia $\mathrm{p}<, 033$, que confirmaría una diferencia del pre y el post test altamente significativa.

También, se considera el grado de instrucción, para comparar las medidas pre y post test (ver tabla 4). 
Tabla 4

Comparación pre test y post test con la prueba $\mathrm{Z}$ de Wilcoxon para el ADCA - I, en adolescentes del $1^{\circ}$ al $5^{\circ}$ año de secundaria $(N=40)$

\section{Análisis pre test - post test}

\begin{tabular}{|c|c|c|c|c|c|c|}
\hline $\begin{array}{l}\text { Grado de } \\
\text { instrucción }\end{array}$ & $\mathbf{N}$ & $\begin{array}{l}\text { Rangos } \\
\text { negativos }\end{array}$ & Empates & $\begin{array}{l}\text { Rangos } \\
\text { positivos }\end{array}$ & $\mathbf{Z}$ & $\begin{array}{c}\text { Nivel de } \\
\text { significación }\end{array}$ \\
\hline $1^{\circ}$ Año & 13 & 4 & 1 & 8 & $-2,278$ & ,023* \\
\hline $2^{\circ}$ Año & 4 & 0 & 0 & 4 & $-1,826$ & ,068 \\
\hline $3^{\circ}$ Año & 13 & 5 & 1 & 7 & $-1,101$ & ,271 \\
\hline $4^{\circ}$ Año & 7 & 3 & 0 & 4 &,- 507 & ,612 \\
\hline $5^{\circ}$ Año & 3 & 0 & 1 & 2 & $-1,342$ & , 180 \\
\hline
\end{tabular}

$* \mathrm{P}<0.05$

En la tabla 4 se observa la comparación pre y post test de alumnos de $1^{\circ}$ a $5^{\circ}$ año de secundaria, de una institución educativa privada del distrito de Ventanilla, con la prueba $\mathrm{Z}$ de Wilcoxon, encontrando diferencias significativas entre el post test y el pre test, de los alumnos de $1^{\circ}$ año de secundaria, con un puntaje $\mathrm{Z}$ de $-2,278$ y $\mathrm{p}<, 023^{*}$, no siendo así con los alumnos de $2^{\circ}$ a $5^{\circ}$ año, donde las diferencias no son significativas.
Así mismo, se toma en cuenta la edad de los participantes, que oscila entre 12, 13, 14 y 15 años de edad, y se comparan las medidas pre y post test, de cada uno; como se muestra en la tabla 5: 
Tabla 5

Comparación pre test y post test con la prueba $\mathrm{Z}$ de Wilcoxon para el ADCA - I, en adolescentes de 12 a 15 años de edad $(N=40)$

\section{Análisis pre test - post test}

\begin{tabular}{lcccccc}
$\begin{array}{l}\text { Grado de } \\
\text { instrucción }\end{array}$ & $\mathbf{N}$ & $\begin{array}{l}\text { Rangos } \\
\text { negativos }\end{array}$ & Empates & $\begin{array}{l}\text { Rangos } \\
\text { positivos }\end{array}$ & $\mathbf{z}$ & $\begin{array}{l}\text { Nivel de } \\
\text { significación }\end{array}$ \\
\hline 12 Años & 11 & 4 & 1 & 6 & $-1,785$ & 0,74 \\
13 Años & 5 & 0 & 0 & 5 & $-2,023$ &, $043^{*}$ \\
14 Años & 11 & 6 & 1 & 4 &,- 153 &, 878 \\
15 Años & 8 & 2 & 0 & 6 & $-1,260$ &, 208 \\
11,16 y 17 Años & 5 & --------- & -------- & -------- & -------- & -------- \\
\hline
\end{tabular}

$* \mathrm{P}<0.05$

* No se consideran las edades de 11,16 y 17 años dada la cantidad pequeña de participantes.

En la tabla 5 se observa la comparación pre y post test de alumnos de 12 a 15 años de edad, de una institución educativa privada del distrito de Ventanilla, con la prueba $\mathrm{Z}$ de Wilcoxon; en la que se distingue que solo los adolescentes de 13 años de edad muestran diferencias significativas entre el post test y el pre test, con un puntaje $\mathrm{Z}$ de $-2,023$ y $\mathrm{p}<, 043$, no dándose esta circunstancia en las otras edades.

Fuera de ello se realizó un análisis con la prueba U de Mann-Whitney al pre test y un mismo análisis al post test, enfrentando a los grupos según género (masculino / femenino) y tipo de familia (biparental / monoparental), con el objetivo de ubicar si existían diferencias significativas entre los resultados de los mismos, luego de haber aplicado el programa de asertividad. Cabe indicar que en cuanto al pre test no se observó diferencias significativas según Tantaleán (2011) - anexo 1. A continuación se muestran los resultados del análisis al post test (ver tabla 6). 
Tabla 6

Comparación de las medidas post test, con la prueba U de Mann-Whitney, para el ADCA - I, en adolescentes del género femenino versus los de género masculino $(\mathrm{N}=40)$

\begin{tabular}{llcccc}
\hline Género & N & Rango promedio & Sumatoria de rangos & U & Nivel de significación \\
\hline Femenino & 27 & 20,54 & 554,50 & 174,500 & 977 \\
Masculino & 13 & 20,42 & & 265,50 & \\
\hline
\end{tabular}

$\mathrm{P}<0.05$

La tabla 6 presenta comparación de las medidas post test, con la prueba U de Mann-Whitney en adolescentes según el género, con una U de 174,500, con $\mathrm{p}<0,977$, confirmando que la diferencia del post test, entre participantes según el género no es significativa.

Tabla 7

Comparación de las medidas post test, con la prueba U de Mann-Whitney, para el ADCA - I, en adolescentes de familias monoparentales versus los de familias Biparentales; $(\mathrm{N}=40)$

\begin{tabular}{lccccc}
\hline Tipo de familia & $\mathbf{N}$ & Rango promedio & Sumatoria de rangos & $\mathbf{U}$ & Nivel de significación \\
\hline Monoparental & 10 & 18,80 & 188,00 & 127,000 &, 931 \\
Biparental & 26 & 18,38 & 478,00 & & \\
$*$ Indistinta & 4 & ------- & -------- & & \\
\hline
\end{tabular}

$* \mathrm{P}<0.05$

* No se considera el tipo de familia indistinta dado que la cantidad de participantes con esta característica es muy pequeña.

La tabla 7 presenta comparación de las medidas post test, con la prueba U de Mann-Whitney en adolescentes según el tipo de familia, con una $U$ de
127,000 y $\mathrm{p}<0,931$, confirmando que la diferencia del post test, entre participantes según el tipo de familia no es significativa. 


\section{DISCUSIÓN}

A partir de los resultados hallados mediante la comparación de medias del pre test y el post test de la variable asertividad para los puntajes obtenidos con la Escala de Evaluación de la Asertividad ADCA - I, se puede afirmar que el programa de asertividad aplicado es efectivo, debido a que produjo un incremento altamente significativo en el desarrollo de conductas asertivas, en un grupo de 40 adolescentes entre 11 y 17 años de edad, estudiantes de una institución educativa privada, del distrito de Ventanilla en la provincia constitucional del Callao.

Por lo tanto esta investigación confirma que el programa "HCTV", tuvo éxito en el desarrollo de asertividad en los adolescentes participantes (logrando adquirir un aprendizaje favorable para la expresión de sus sentimientos, emociones, ideas y necesidades).

Es importante recalcar que este estudio no se basa en un solo enfoque, dando paso a una integración de varios modelos, tales como: el modelo cognitivo conductual (al tratar de ubicar ideas erróneas), el modelo del aprendizaje social (cuando se utiliza el modelado) y el modelo conductual (en el trabajo en base al refuerzo, y la retroalimentación); modelos reconocidos por su eficacia tanto por Hidalgo y Abarca (1995, citado en Oblea, 2000), como por Arons (1994, citado en Espino, 1997). Ello se puede afirmar debido a que el programa se presta al uso de las técnicas básicas del entrenamiento asertivo las cuales tienen influencia de más de una tendencia.

Cabe indicar que la efectividad de este programa sobrelaasertividad-sepuedeasociaradiversosaspectos: en primer lugar, el programa logra representar a la variable evaluada, en las diversas temáticas que supone la adquisición de la competencia; en segundo lugar a su estructura de aplicación ceñida al entrenamiento básico en habilidades sociales (instrucción, modelado, ensayo conductual, retroalimentación, refuerzo), las cuales son el punto de partida para el entrenamiento asertivo además de las técnicas de reestructuración cognitiva implementadas por Caballo (1991, citado en Paula, 2000), las técnicas asertivas para afrontar discusiones propuestas por Castanyer (2007) y las técnicas de comunicación asertiva descritas por Navas (1987). En tercer lugar al público objetivo que conformó la muestra de la investigación (adolescentes, quienes por sus características naturales, enfrentan en la vida cotidiana la oportunidad para responder a sus dificultades con conductas asertivas o no asertivas, dado esto en el trato con sus padres, hermanos, compañeros de clase, similares entre otros), brindándoles así las herramientas necesarias para crecer en diversos aspectos de su comunicación interpersonal; y finalmente en cuarto lugar, a la relación íntima que se generó durante el programa, logrando un trabajo personalizado, participativo y continuo, ampliamente reforzado por el aliento de parte del entrenador y los compañeros del taller (al preocuparse por sus derrotas, festejar sus victorias; comprender sus temores cuando alguno reconocía sus carencias e intentaba cambios concretos), dando a este espacio un clima familiar y de confianza.

Los resultados hallados son similares a los obtenidos por Garaigordobil (2001, en Garaigordobil, 2006), quien diseñó un programa de intervención grupal para adolescentes relacionado con el desarrollo de conductas asertivas, evaluando sus efectos en variables conductuales y cognitivas de la interacción social, con un diseño pre test intervención post test con grupos de control, en una muestra constituida por 174 adolescentes de 12 a 14 años de edad, encontrando un impacto positivo del programa, confirmando un incremento significativo de la auto-asertividad, de las conductas sociales asertivas, de las conductas de liderazgo y de las estrategias cognitivas asertivas de solución de situaciones sociales conflictivas, así como una disminución de las conductas de ansiedadtimidez; los datos además sugieren que la experiencia fue especialmente significativa para los adolescentes con bajo nivel de desarrollo social en el pre test. Por su parte Pinares (2006), quien realizó una investigación cuasi experimental, con el propósito de conocer los efectos de un conjunto de técnicas participativas sobre las habilidades sociales en adolescentes trabajadores entre 12 y 17 años de edad, logró un resultado significativo, comprobando a su vez que las técnicas de reestructuración cognitiva entre otras asociadas con las técnicas participativas son eficaces para asumir un cambio comportamental; al igual que Choque (2007), quien buscó determinar la eficacia de un programa educativo de habilidades para la vida en el aprendizaje de las habilidades de comunicación, autoestima, asertividad y toma de decisiones, en adolescentes escolares de una institución educativa del 
distrito de Huancavelica, encontrando un incremento significativo en las habilidades de comunicación y asertividad en los estudiantes del grupo experimental en comparación a los del grupo control, concluyendo así que el programa propuesto, es efectivo para las variables antes mencionadas.

Por otra parte, tanto el programa como la prueba utilizada para medir su efectividad están íntimamente relacionados, por ejemplo en el listado de los derechos asertivos y los elementos que mide el ADCA-1, presentado por García y Magaz (1995), representando así aspectos básicos que forman parte del programa "HCTV", según su temática.

Fuera de ello existen otras apreciaciones al respecto, como indica Capa (1997), quien asume que las variables pueden elevarse o decrecer, no interesa el sistema de evaluación, ni el estilo de desarrollo, pero sí los resultados de los cuales se pueden obtener conclusiones concretas. Este autor, para efectos de su investigación referente a la relación entre conducta asertiva y rendimiento en estudiantes de secundaria, utilizó el modelo interconductual, como base, midiendo la variable asertividad mediante un inventario de corte conductual, obteniendo resultados favorables. Cabe indicar que este aspecto puede llevar hacia una polémica, la cual no será necesaria, puesto que existe concordancia en todos los aspectos que desarrolla esta investigación.

Es importante tomar en cuenta los resultados hallados por el análisis estadístico de comparación pre y post test, según grado de instrucción, con mejoras significativas en la conducta asertiva de los alumnos de menor grado de instrucción, ello a pesar que inicialmente se consideró que los alumnos de grados superiores tenían mayor ventaja para asimilar los contenidos del programa; sobre ello es básico valorar que la motivación es un elemento muy importante para la consecución de objetivos, puesto que fueron los participantes de $1^{\circ}$ año de secundaria los que se mostraron más entusiastas, denotándolo tanto en su perseverancia como en la presentación de sus tareas prácticas, sesión a sesión. Según la edad los resultados son parecidos, denotando mayor efectividad del programa en alumnos de 13 años de edad; conduciendo a deducir que a mayor edad, mayor dificultad para el cambio; puesto que al pasar el tiempo entre los 14 y 17 años los estudiantes suelen aumentar sus expectativas de vida, aumentado sus intereses y diversificándolos. A manera de ejemplo se puede utilizar un detalle característico de la muestra de este estudio, quienes a partir de los 14 años, ya habían iniciado una relación de pareja (el 65\% de los participantes del programa), lo que representaría un distractor considerable, entre otros; conclusión semejante a la de Guerrero y Lizano (1998), quienes afirman que entre los 13 y 14 años se da un periodo de crisis -en la adolescencia-, produciéndose cambios profundos en el carácter que tienden a desencadenar conflictos; no sucediendo con las otras edades, que denotan cierta estabilidad (como punto de quiebre en el desarrollo de su autoasertividad).

Con respecto a las variables sexo y tipo de familia. se intentó observar cuáles eran las implicancias del programa al finalizar su aplicación y quiénes denotaban mayores cambios en la asertividad; podría haberse asumido que en cuestión al género, serían los participantes de género femenino quienes logren mayores cambios, como lo indica Garaigordobil (2006) en su estudio sobre las relaciones existentes entre el autoconcepto, autoestima, sociabilidad, estabilidad emocional y responsabilidad en adolescentes entre los 14 y 17 años, investigación en la cual las mujeres obtienen mayor puntaje que los varones, en habilidades para la comunicación; o deducir que los provenientes de familias biparentales, se encuentran en mayor ventaja para asimilar la asertividad que los que pertenecen a familias monoparentales, como lo indica Mescua (2009), quien en su investigación sobre la relación entre el nivel de autoestima y el tipo de familia de procedencia de estudiantes de enfermería, concluyó que el tipo de familia era un aspecto importante para el desenvolvimiento socio emocional, indicando que, en su mayoría, las participantes evaluadas que poseían un comportamiento acorde al perfil de la profesión, provenían de familias articuladas y funcionales.

Sobre los dos aspectos antes mencionados, es importante acotar que aunque ambos grupos considerados según el género y/o tipo de familia partieron de circunstancias similares en la evaluación pre test con la prueba de U de Mann-Whitney, no variaron considerablemente en el post test.

En cuanto al sexo se puede asumir, según Guerrero y Lizano (1998), que los cambios bruscos que asimilan tanto varones como mujeres en su sexualidad, los 
coloca en situación similar, dado que son espectadores de todo cuanto sucede en su interior (cambios físicos y hormonales), como lo que sucede en su exterior (posicionamiento en su medio social).

Para finalizar, es importante acotar que cada individuo posee elementos internos que coadyuvan o no a la asimilación de una competencia, por tanto el factor de auto-motivación, tiende a ser el punto central del cual parte todo cambio. Ello tomando en cuenta a los participantes del programa (varones o mujeres) y/o con estructura familiar (monoparental o biparental), tuvieron el deseo de desarrollar su asertividad, por el solo hecho de asistir con puntualidad e interés a las 8 sesiones propuestas.

Al finalizar el estudio se concluye que:

- Se encontraron diferencias significativas en la asertividad, pre y post test; luego de aplicar el programa: "HCTV", en adolescentes de una institución educativa privada de Ventanilla Callao.

- Solo se encontraron diferencias significativas en la asertividad, pre y post test, luego de aplicar el programa "HCTV" en adolescentes del primer año de secundaria de una institución educativa privada de Ventanilla - Callao, no siendo así con los años académicos superiores.

- Solo se encontraron diferencias significativas en la asertividad, pre y post test; luego de aplicar el programa "HCTV" en adolescentes de 13 años de edad, estudiantes de una institución educativa privada de Ventanilla - Callao, no siendo así con los de otras edades.

- No se descubrieron diferencias significativas en la asertividad, pos test; luego de aplicar el programa "HCTV" entre adolescentes de género masculino y femenino, estudiantes de una institución educativa privada de Ventanilla - Callao.

- No se descubrieron diferencias significativas en la asertividad, post test; luego de aplicar el programa "HCTV" entre adolescentes provenientes de familia monoparental y biparental, estudiantes de una institución educativa privada de Ventanilla - Callao.
Al finalizar el estudio se recomienda:

- Extender el estudio a adolescentes en situación de riesgo.

- Aplicar el programa en una muestra que presente considerable déficit en asertividad.

- Adaptar el programa para aplicarlo a niños, adolescentes (según su nivel socio-económico y socio cultural).

- Crear un módulo de 4 sesiones para padres de familia, docentes y administrativos de los centros educativos; con la finalidad de extender la variable asertividad, dentro de la cultura organizacional de la escuela.

- Incorporar estos talleres a la estructura curricular del curso persona familia y relaciones humanas, en los centros de estudio secundario y asumir un modulo de desarrollo de habilidades para la comunicación en centros de instrucción superior y técnica.

- Elaborar un instrumento que evalúe al detalle las competencias que se desarrollan en el programa "HCTV".

\section{REFERENCIAS}

Calle, M. (2005). Modelo de atención integral en salud-etapa adolescente. Lima, Perú: Ministerio de Salud (MINSA).

Capa, W. (1997). Relación entre conducta asertiva $y$ rendimiento en estudiantes de secundaria de nivel socioeconómico medio-alto (Tesis de Licenciatura no publicada). Universidad Nacional Federico Villarreal, Lima.

Castanyer, O. (2007). La asertividad expresión de una sana autoestima. Bilbao: Descleé de Brouwer.

Choque, R. (2007). Evaluación del programa educativo de habilidades para la vida en educación secundaria (Tesis de Maestría no publicada). Universidad Cayetano Heredia, Lima. 
Espino, R. (1997). Habilidades sociales en niños y adolescentes. Monografía no publicada, para optar el título de Licenciado. Universidad Nacional Federico Villarreal, Lima.

Fábregas, J. y García, E. (1988). Técnicas cognitivas de autocontrol. Madrid: Alhambra,

Garaigordobil, M. (2006). Relaciones del autoconcepto y la autoestima con la sociabilidad, estabilidad emocional y responsabilidad en adolescentes de 14 a 17 años. Análisis y Modificación de Conducta, 32, 37-64.

García, M. y Magaz, A. (1995). Agresividad y retraimiento social: el entrenamiento en habilidades sociales. Madrid: Arbor.

Guerrero, M. y Lizano, C. (1998). Test de autoinforme de conducta asertiva ADCA-1 en adolescentes de 12 a 17 años de edad baremado en Lima Metropolitana (Tesis de Licenciatura no publicada). Universidad Femenina del Sagrado Corazón, Lima.

Hernández, R., Fernández, J. y Baptista, P. (2006). Metodología de la Investigación. México D.F.: McGraw-Hill.

Instituto Nacional de Estadística e Informática (2000). Encuesta demográfica y de salud familiar. Perú.

Instituto Nacional de Estadística e Informática (2011). Estimaciones y proyecciones de la población, según grupos quinquenales de edad, 2010-2025. Perú.

Jara, E. (1997). Asertividad: concepciones, evaluación y programas de entrenamiento. Monografía no publicada, para optar el título de Licenciado. Universidad Nacional Federico Villarreal, Lima.

Lange, A. (1981). Entrenamiento cognitivo conductual de la asertividad. En Ellis, A. Manual de TRM. Bilbao: Descleé de Brower.

Fecha de recepción: 2 de noviembre de 2012

Fecha de aceptación: 7 de diciembre de 2012
Mescua, G. (2009). Relación entre el nivel de autoestima y el tipo de familia de procedencia de los estudiantes de enfermería de la Universidad Nacional Mayor de San Marcos (Tesis de Licenciatura no publicada). Universidad Nacional Mayor de San Marcos, Lima.

Montgomery, W. (1997). Asertividad, autoestima y solución de conflictos interpersonales. Lima: Círculo de Estudios Avanzada.

Navas, J. (1987). Como tomar decisiones y solucionar problemas racionalmente. Santo Domingo: Librotex.

Oblea, V. (2000). Programas de entrenamiento en habilidades sociales. Monografía no publicada, para optar el título de Licenciado. Universidad Nacional Federico Villarreal, Lima.

Olano, S. y Risco, G. (2005). Clima motivacional de la clase, asertividad y rendimiento académico de los alumnos de la Universidad Cesar Vallejo. Revista de Psicología de la Universidad César Vallejo, 7, 97-114.

Paula, I. (2000). Habilidades sociales educar hacia la autorregulación. Barcelona: Cuadernos de educación.

Pinares, D. (2006). Técnicas participativas y habilidades sociales en adolescentes trabajadores de Huaycán (Tesis de Licenciatura no publicada). Universidad Nacional Federico Villarreal, Lima.

Tantaleán, L (2011). Efectos de un programa "Hazte Cargo de tu Vida", en la asertividad de adolescentes de una institución educativa privada de Ventanilla - Callao (Tesis de Maestría no publicada). Universidad Femenina del Sagrado Corazón, UNIFÉ, Lima, Perú.

Quinn, M., Quinn, T. (2002). Programa "Hazte cargo de tu vida". Lima: Centro Catequético Salesiano. 